\title{
Poster Sessions
}

MS16.P16

Acta Cryst. (2011) A67, C292
MS16.P17

Acta Cryst. (2011) A67, C292
Reversible DNA scission by type II topoisomerase from Streptococcus pneumoniae and its modification upon drug binding

Ivan Laponogov, ${ }^{\mathrm{a}, \mathrm{b}}$ Xiao-Su Pan, ${ }^{\mathrm{b}}$ Dennis A. Veselkov, ${ }^{\mathrm{a}}$ Katherine McAuley, ${ }^{c}$ L. Mark Fisher, ${ }^{b}$ Mark R. Sanderson ${ }^{\mathrm{a}} \quad{ }^{a}$ Randall Division of Cell and Molecular Biophysics, King's College London, 3rd Floor, New Hunt's House, Guy's Campus, University of London, London, (UK). ${ }^{b}$ Molecular Genetics Group, Molecular and Metabolic Signalling Centre, Department of Basic Medical Sciences, St. George's, University of London, London, (UK). 'Diamond Light Source, Didcot, Oxford, (UK). E-mail: ivan.laponogov@kcl.ac.uk, mark.sanderson@kcl.ac.uk, lfisher@sgul.ac.uk

Type II topoisomerases are essential enzymes responsible for changing and stabilizing DNA supercoiling and are also involved in chromosome segregation in prokaryotes. They are also targeted by antibacterials such as quinolones or quinazolinediones. Topo II enzymes operate by forming a transient covalent cleavage complex with a gate-DNA (G-segment) duplex and transporting the second duplex (T-segment) though a double-stranded break in the formed protein-DNA gate thus changing the DNA supercoiling in steps of 2. Despite the biological and clinical importance of these enzymes, the current understanding of their mechanism of action and enzymeantimicrobial drug interaction. In order to further our understanding of the topo II role in DNA supercoiling and drug interaction, we have solved several crystal structures of a DNA cleavage complex of topoisomerase IV from $S$. pneumoniae stabilised by a number of different antibacterials and also captured in-crystal two stages of the cleavage complex reversal thus providing the structural snapshots the sequential states of enzyme operation[1], [2].

Drug-arrested forms of the cleavage complex were solved in presence of quinazolinedione PD 0305970 (3.1 $\AA$ ) and quinolones such as levofloxacin $(2.9 \AA)$ and clinafloxacin $(3.35 \AA)$. In all studied cases the structures revealed two drug molecules intercalated at a symmetrically cleaved B-form DNA gate and stabilized by drugspecific protein contacts. In contrast with the dione-stabilised cleavage complex the quinolone-stabilised complexes contain magnesium ions chelated by the drug molecules and involved in protein-drug interaction which explains the magnesium dependence of the quinolone activity.

Drug-free cleaved and resealed DNA complexes were obtained from the original dione-stabilised cleavage complex by subsequent dione release. In contrast with the drug-stabilised complexes the DNA gate in drug-free complexes adopts an unusual A/B-form helical conformation and the active site magnesium ion is positioned close to the scissile phosphodiester group thus allowing its coordination and promoting reversible cleavage by active-site tyrosines. These structures solved for putative reaction intermediates of a type II topoisomerase provide the structural information about different stages of a normal reaction cycle of the enzyme as well as drug-specific interaction mechanisms which is important for the rational drug design in the future.

[1] I. Laponogov, M.K. Sohi, D.A. Veselkov, X.-S. Pan, R. Sawhney et al. Nature Structural Molecular Biology 2009, 16, 667-669. [2] I. Laponogov, X.-S. Pan, D.A. Veselkov, K.E. McAuley, L.M. Fisher, M.R. Sanderson, PLoS ONE 2010, 5(6), e11338.

Keywords: topoisomerases, breakage-reunion, protein-DNA complexes
Structure and molecular interactions of an anti-tumor antibody specific for EGFR

Ariel Talavera, ${ }^{\mathrm{a}}$ Rosmarie Friemann, ${ }^{\mathrm{d}}$ Silvia Gómez-Puerta, ${ }^{\mathrm{a}}$ Carlos Martinez-Fleites, ${ }^{\mathrm{c}}$ Greta Garrido, ${ }^{\mathrm{a}}$ Ailem Rabasa, ${ }^{\mathrm{a}}$ Alejandro LopezRequena, ${ }^{\mathrm{a}}$ Amaury Pupo, ${ }^{\mathrm{a}}$ Rune F. Johansen, ${ }^{\mathrm{d}}$ Oliberto Sánchez, Ute Krengel, ${ }^{b}$ Ernesto Moreno, ${ }^{a}$ Center of Molecular Immunology, Havana, Cuba. ${ }^{b}$ Dept. of Chemistry, University of Oslo, Oslo, Norway. ${ }^{c}$ Dept. of Chemistry, University of York, York, (UK). ${ }^{d}$ Institute of Medical Microbiology, University of Oslo, Oslo, Norway. ${ }^{e}$ Center for Genetic Engineering and Biotechnology, Havana, Cuba. E-mail: talavera@cim.sld.cu

Overexpression of the epidermal growth factor (EGF)receptor (EGFR) in cancer cellscorrelates with tumor malignancy and poor prognosis for cancer patients. For this reason, the EGFR has become one of the main targets of anti-cancer therapies. Structural data obtained in the last few years have revealed the molecular mechanism for ligand induced EGFR dimerization and subsequent signal transduction, and also how this signal is blocked by either monoclonal antibodies or small molecules.

Nimotuzumab (also known as h-R3) is a humanized antibody that targets the EGFR and has been successful in the clinics. In this work we report the crystal structure of the Fab fragment of Nimotuzumab, revealing some unique structural features in the heavy variable domain. Furthermore, competition assays show that Nimotuzumab to domain III of the extracellular region of the EGFR, within an area that overlaps with both the surface patch recognized by Cetuximab (another antiEGFR antibody) and the binding site for EGF. A computer model of the Nimotuzumab-EGFR complex, constructed by docking and molecular dynamics simulations and supported by mutagenesis studies, unveils a completely novel mechanism of action, with Nimotuzumab blocking EGF binding while still allowing the receptor to adopt its active conformation, hence warranting a basal level of signaling.

Keywords: cancer, egfr, antibody

\section{MS16.P18}

Acta Cryst. (2011) A67, C292-C293

Saquinavir resistance mechanism of non-active site mutants of HIV-1 Protease

Vishal Prashar, ${ }^{\text {a }}$ Subhash Bihani, ${ }^{\text {a }}$ Amit Das, ${ }^{\text {a }}$ Jean-Luc Ferrer, ${ }^{\text {b }}$ Madhusoodan Hosur, ${ }^{a}$ B Bhabha Atomic Research Centre, Solid State Physics Division, V.N.Purav Marg, Trombay, Mumbai, Maharashtra, 400085, India, 'Institut de Biologie Structurale (IBS), CEA/CNRS/ UJF, Grenoble Cedex 1, France. E-mail: vishalp@barc.gov.in

Since virally encoded protease plays a key role in the life cycle of Human immunodeficiency virus (HIV-1), it is the prime target of drugs in chemotherapy against Acquired immunodeficiency syndrome (AIDS). Protease inhibitors are important components of Highly Active Anti Retroviral Therapy (HAART) which is a standard treatment regimen followed for the treatment of HIV/AIDS. However, emergence of drug-resistant mutations in HIV-1 protease necessitates continuous improvement of existing drugs and design of new HIV1 protease inhibitors based on understanding of the mechanisms of drug resistance. Structurally, these mutations can be categorized into active and non-active site mutations. As all the approved anti-HIV-1 protease drugs are competitive inhibitors, active site mutations may directly affect their binding. But effect of non-active site mutations is indirect and difficult to comprehend. Saquinavir (SQV) is one of 